\title{
Mean Velocity of Raindrops at Orthogonal Polarizations of Radar Sounding Waveform
}

\author{
Dmitry Glushko, Felix J. Yanovsky \\ Radioelectronics Department, National Aviation University \\ Prospect Komarova 1, Kiev, 03680, Ukraine \\ dima-pilot@mail.ru \\ yanovsky@i.com.ua
}

\begin{abstract}
ABSTARCT
This paper studies Doppler spectra of the reflections from rain at different polarizations. Particularly, it presents results of Doppler-polarimetric radar signal modelling and real data processing that show correlation between Differential Doppler Velocity (DDV), which is the difference between mean Doppler velocities at horizontal and vertical polarizations, and parameters of rain including rain microstructure and turbulence in rain.
\end{abstract}

\section{INTRODUCTION}

A model calculating Doppler-polarimetric spectra of returns from rain was developed in 1996-97 and verified using Delft Atmospheric Research Radar (DARR) in 1998 [1]. An important feature of that model consists in taking into account inertia of raindrops. Bigger drops are more oblate, they fall faster and contribute more to the reflectivity, but they are more inertial and less involved into turbulent motion than small droplets. Kolmogorov-Obukhov model of turbulence assumes constant eddy dissipation rate in the inertial sub-range. The model [1] uses Kolmogorov-Obukhov expression, assuming that some spatial scale of turbulence exists, which impels the droplets of less than given size to repeat the turbulent motion. All bigger turbulent scales do the same while smaller ones cannot influence to the droplets of given or bigger size. This is a kind of simplification, which allows taking into account the inertia of droplets which depends on droplet size. Such approach was borrowed from [2]. Using this approach, different Doppler-polarimetric parameters were investigated, for example, the spectral differential reflectivity [3] that is related with turbulence intensity. Differential Doppler velocity (DDV) was introduced in [4] and [5] by a little bit different ways. In particular, the definition [4] tries to exclude the dependency of DDV upon antenna elevation $\theta$ while definition [5], [6] keeps this dependence, producing the calculations at different values $\theta$. As a matter of fact, the idea of both ways is the same.

Estimation and especially interpretation of Doppler-polarimetric measurable variables require rather sophisticated signal processing algorithms. Clear understanding of physics and statistics of the relationships between Doppler-polarimetric measurands and parameters of microstructure and dynamic processes of weather objects is important for the development of signal processing algorithms. Another approach is related with fuzzy logic and neural network approaches that give possibilities to avoid bulky computations and time losses during signal processing while they can take into account many significant measurands applying the last scientific achievements into the practice. These approaches were successfully applied to such kind of tasks in [7] for hydrometeor type classification and in [8] for both hydrometeor type and turbulence intensity determination. In this aspect, additional informative parameters are always desirable especially if they carry independent information on the features of the object under study and may increase the functionality, accuracy and reliability of the system.

In this paper we study a new Doppler-polarimetric parameter - differential Doppler velocity (DDV) in more detail. Our goal is to show that this parameter is associated with both rain microstructure and turbulence in rain and may be useful in meteorological radar as instrument for hydrometeor type recognition and turbulence intensity estimation.

\section{DOPPLER-POLARIMETRIC MESURANDS}

There are a number of measurands typical for polarimetric weather radar, such as differential reflectivity $Z D R$, linear depolarization ratio $L D R$, copolar correlation coefficient between orthogonally polarized signals $\rho$, specific differential

Photonics Applications in Astronomy, Communications, Industry, and High-Energy Physics Experiments 2009, edited by Ryszard S. Romaniuk, Krzysztof S. Kulpa, Proc. of SPIE Vol. 7502, 750211 - () 2009 SPIE · CCC code: 0277-786X/09/\$18 - doi: 10.1117/12.838055 
phase $K d p$ [7]. Some new measurable characteristics were studied in [2], particularly spectral differential reflectivity was

defined as $s Z d r=10 \lg \left[S_{h h}(v) / S_{v v}(v)\right]$ with $S_{h h}(v)$ and $S_{v v}(v)$ as Doppler spectra estimations at horizontal $(h h)$ and vertical $(v v)$ polarizations.

DDV parameter was defined as $\Delta V=\bar{V}_{h h}-\bar{V}_{v v}$, where $\bar{V}_{h h}$ and $\bar{V}_{v v}$ are mean Doppler velocities at horizontal and vertical polarization correspondingly. In rain the bigger drops are more oblate and they fall faster than smaller raindrops. Moreover, bigger drops contribute more to the value of mean Doppler velocity at horizontal polarization than at vertical polarization due to specific primary orientation in space. That is why components of the Doppler spectrum at horizontal polarization is a little bit bigger than ones at vertical polarization, and this difference depends on microstructure of rain and the order of abnormality of droplets orientation that can occur due to the turbulence. That means that DDV in rain should be positive.

\section{THEORETICAL CONSIDERATION}

The basic initial models which are necessary for theoretical consideration of DDV are the models of: a) microstructure of scatterers in resolution volume of rain; b) turbulence that may affect the scatterers; c) inertia of raindrops, which do not follow turbulence motion perfectly; d) coordinates and polarization basis.

The microstructure of rain in described by generally accepted Gamma dropsize distribution in the form [6]

$$
N(D)=N_{0} D^{\mu} \exp \left(-\frac{3.67+\mu}{D_{0}} D\right)
$$

where $N(D)$ is the number of droplets with an equivalent diameter between $D$ and $D+d D$ in the unit of volume, $D_{0}$ is the diameter of the median drop volume. Expression (1) includes the Marshall-Palmer model as a particular case for $\mu=0$. In case $\mu \neq 0, N_{0}$ can be derived [6] from the Marshall-Palmer distribution by keeping the rainwater content constant for a given $D_{0}$. The fall speed of raindrops in stagnant air is related to their size. The relationship between drop diameter $D$ and the drop fall speed $v_{f}$ is given in [9]:

$$
v_{f}(D)=9.65-10.3 \cdot e^{-0.6 D}, \quad D \geq 0.109[\mathrm{~mm}] .
$$

The shape of a droplet is described by the axial ratio $\rho=a_{3} / a_{1}$, where $a_{1}$ and $a_{3}$ horizontal and vertical semi-axes of the spheroid as a model of droplet shape, and following [6]

$$
\rho(D)=\frac{1}{2}\left[\exp \left(-\frac{D^{2}}{27}\right)+1\right]
$$

where $D$ is equivolumetric droplet diameter in $\mathrm{mm}$.

Turbulence is modeled by its energetic spectrum according to Kolmogorov minus five third law:

$$
S(\Omega)=C \varepsilon^{2 / 3} \Omega^{-5 / 3},
$$

where $\varepsilon$ is the eddy dissipation rate, $C$ is dimensionless constant, $\Omega$ is spatial frequency $\Omega=2 \pi / L$ with $L$ as turbulence vortex scale, a random value. Inertia of droplets was taken into account following [3].

Linear orthogonal polarization basis was used. Polarization of radar signal depends on antenna orientation. "Horizontal" polarization $(\phi=0)$ is really horizontal (E-vector lies in the horizontal plane independently on elevation angle $\theta$ ); position of vector $\mathrm{E}$ at "vertical" polarization $(\phi=\pi / 2)$ depends on elevation angle $\theta$ : it is really vertical at horizontal sounding, it is horizontal at the sounding into zenith. 
Considering Doppler-polarimetric approach one should take into account that Doppler measurements give the information about radial velocities of the hydrometeors while polarimetry is connected with the shape and orientation of the hydrometeors. In frequency domain Doppler-polarimetric radar is able to measure Doppler energy spectra at different combinations of polarizations of transmitted and received waves, so called Doppler-polarimetric spectra.

Theoretically, Doppler-polarimetric spectra can be calculated as

$$
S_{m n}(v / \varepsilon, \theta)=\int_{D_{\min }}^{D_{m n}} p(v / D, \varepsilon, \theta) \sigma_{m n}(D / \theta, \varepsilon) N\left(D / D_{o}\right) d D,
$$

where $p(v / D, \varepsilon, \theta)$ is velocity distribution of a droplet with diameter $D$ which is moving due to gravity and influence of wind (turbulence);

$\sigma_{m n}(D / \theta, \varepsilon)$ is RCS of the non-spherical droplet of given equivolumetric diameter at $\theta$ antenna elevation and $\varepsilon$ turbulence intensity (eddy dissipation rate) for $m n$ polarization $(m=h ; v, n=h ; v)$. In case $\lambda \gg>$ RCS is

$$
\sigma_{m n}=\frac{\pi^{5} D^{6}}{9 \lambda^{4}}\left|\varepsilon_{r}-1\right|^{2} F_{m n}(\Lambda) \Phi_{m n}(\delta, \alpha, \theta)
$$

where $F_{m n}(\Lambda)$ and $\Phi_{m n}(\delta, \alpha, \theta)$ represent the shape of the particle (quantified with $\Lambda$ ) and orientation $(\delta, \alpha)$ of the droplet; $\varepsilon_{r}$ characterizes electromagnetic permeability.

Probability density of droplet velocity caused by turbulence can be calculated as done in [5]:

$$
p_{T}\left(v_{T} / D, \varepsilon\right)==\int_{L_{\text {thres }}(D)}^{L_{\max }} w_{T}\left(v_{T} / L, \varepsilon\right) w(L) d L
$$

with $w_{T}$ as probability density of turbulence velocity and $w(L)$ as probability density of spatial scales of turbulence. Integration over $D$ gives DTVD - droplet turbulence velocity distribution of an ensemble of droplets:

$$
N_{T}\left(v_{T} / \varepsilon\right)=\int_{D_{\min }}^{D_{\max }} p_{T}\left(v_{T} / D, \varepsilon\right) N(D) d D=\int_{0}^{\infty} p_{T}\left(v_{T}, D\right) N(D) d D .
$$

Velocity distribution $p(v)$ caused by both turbulence $v_{T}$ and gravity $v_{f}$ can be calculated if one takes into account that $v=v_{f}+v_{T}$ and partial distribution (for specific droplet diameter) can be written as $p_{p}(v / D)=p_{T}\left[\left(v-v_{f}\right) / D\right]$. After integration over all droplet diameters one can get distribution summarized distribution:

$$
p_{\Sigma}(v)=\int_{o}^{D m} p_{T}\left\{\left[v-v_{f}(D, \theta)\right] / D\right\} N(D) d D
$$

and desired probability density

$$
p(v)=p_{\Sigma}(v) / \int_{-\infty}^{\infty} p_{\Sigma}(v) d v
$$

Finally, Doppler-polarimetric spectra are calculated by substitution (10), (6) and (1) into expression (5), and then mean Doppler velocities are derived by 


$$
\bar{V}_{h h}=\int_{-\infty}^{\infty} v S_{h h}(v) d v ; \quad \bar{V}_{v v}=\int_{-\infty}^{\infty} v S_{v v}(v) d v .
$$

This technique ensures the calculation of DDV at different conditions because DDV $=\bar{V}_{h h}-\bar{V}_{v v}$ appear as a positive value that is a function of antenna elevation angle $\theta$, eddy dissipation rate $\varepsilon$, and parameters of Gamma dropsize distribution: median drop diameter $D_{0}$ and spread $\mu$.

\section{CULCULATION AND SIMULATION RESULTS}

Examples of non-normalized Doppler spectra of rain calculated at horizontal and vertical polarizations at the same parameters of microstructure are shown in Fig. 1, 2 and 3. The calculations were done at the following initial data: antenna elevation angle $\theta=45$ degrees; parameters of Gamma dropsize distribution $\mu=2, D_{0}=2, \mathrm{~N}_{0}=8000$. The turbulence intensity given by eddy dissipation rate $\varepsilon$ was changed ten times from case to case: $\varepsilon=0.1, \varepsilon=1$, and $\varepsilon=10\left[\mathrm{~cm}^{2} / \mathrm{s}^{-3}\right]$. All these values of $\varepsilon$ characterize different levels of negligible or light turbulence.

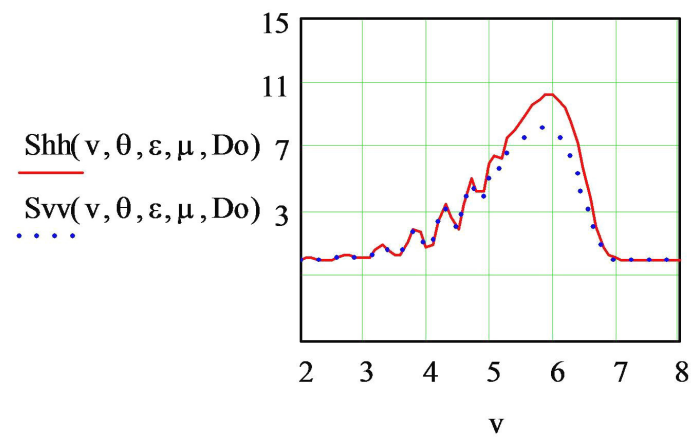

Fig.1. Doppler spectra at horizontal (solid curve) and vertical polarization (dotted curve). $\varepsilon=0.1 \mathrm{~cm}^{2} \mathrm{~s}^{-3}$.

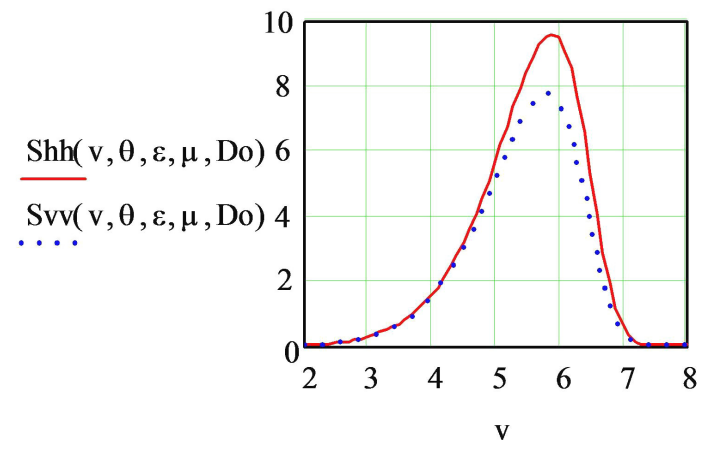

Fig.2. Doppler spectra at horizontal (solid curve) and vertical polarization (dotted curve). $\varepsilon=1 \mathrm{~cm}^{2} \mathrm{~s}^{-3}$.

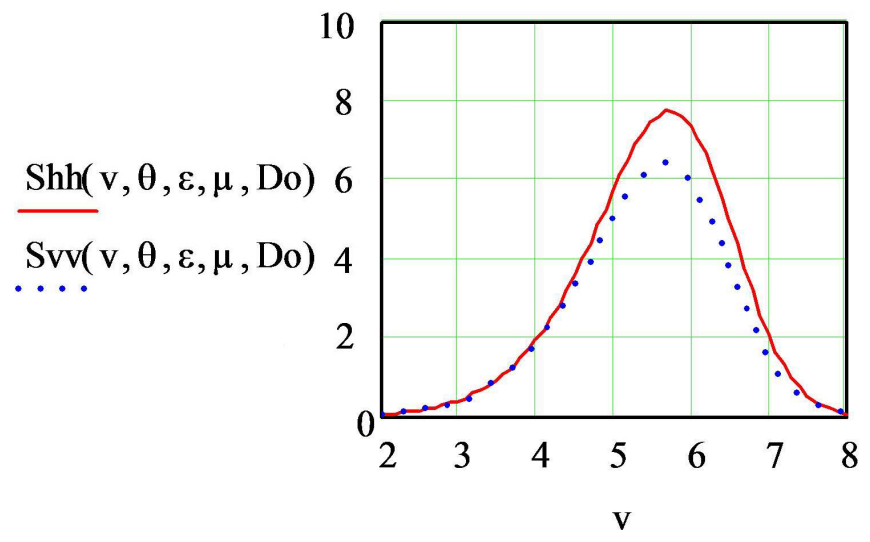

Fig.3. Doppler spectra at horizontal (solid curve) and vertical polarization (dotted curve). $\varepsilon=10 \mathrm{~cm}^{2} \mathrm{~s}^{-3}$. 
One can see that spectral components at horizontal polarization have bigger magnitudes than at vertical polarization as was predicted. Maximum value becomes less and spectrum is wider with increasing turbulence intensity.

Then the behavior of DDV parameter was investigated under the different conditions. Particularly, DDV values as function of eddy dissipation rate and similar microstructure parameters are presented in Fig. 4.

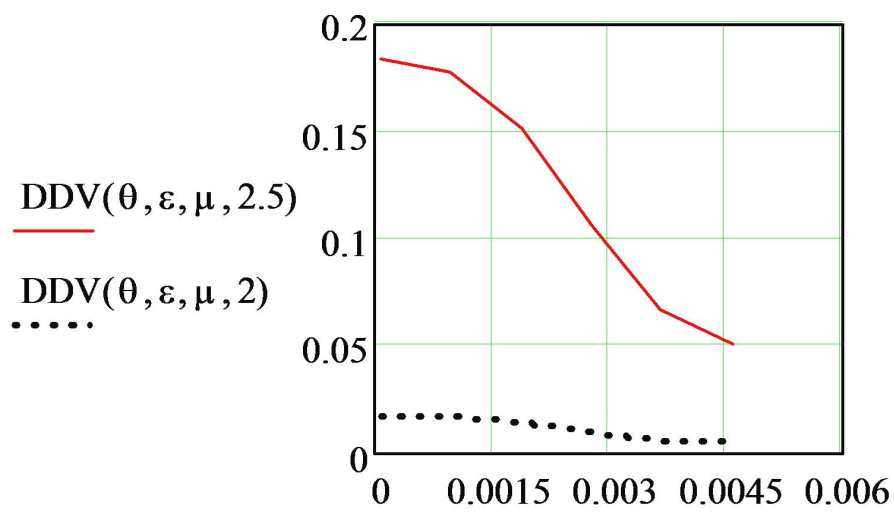

$\varepsilon$

Fig.4. DDV curves as function of eddy dissipation rate $\varepsilon\left[\mathrm{m}^{2} \mathrm{~s}^{-3}\right]$. Solid curve corresponds to $D_{o}=2.5 \mathrm{~mm}$ and dotted curve corresponds to $D_{o}=2.0 \mathrm{~mm}$.

It is seen from the figure that under the action of turbulent air motions, DDV decreases with increasing eddy dissipation rate. Median drop diameter $D_{o}$ is strongly related with intensity of rain, and larger droplets provide higher DDV values. Next example (Fig.5) shows dependence between DDV and one of the important microstructure parameter - spread of Gamma dropsize distribution $\mu$.

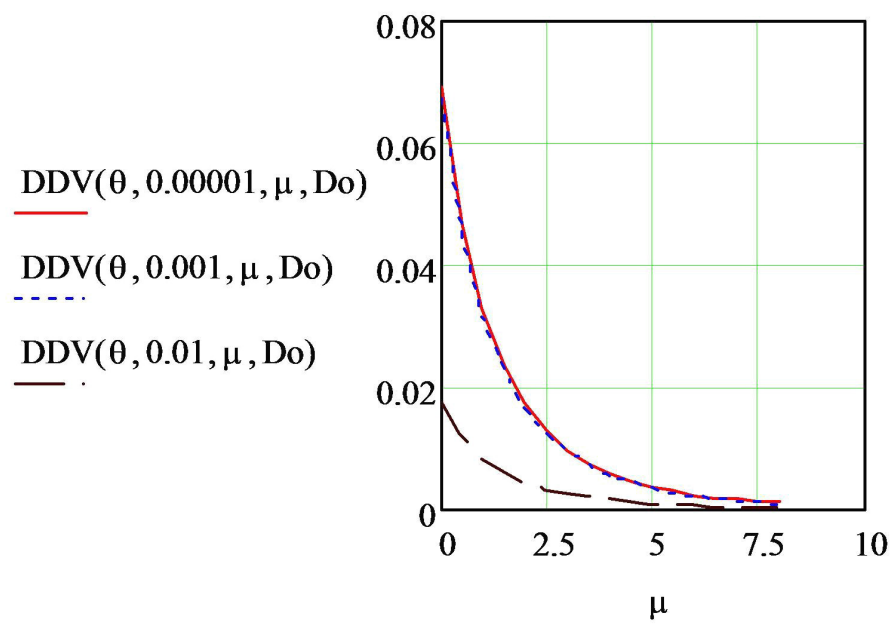

Fig.5. DDV curves as function of spread parameter $\mu$ of Gamma dropsize distribution model. Calculations were done at $D_{o}=2.0 \mathrm{~mm}$ and three values of eddy dissipation rate $\varepsilon$ indicated as numbers in $\left[\mathrm{m}^{2} \mathrm{~s}^{-3}\right]$. Solid curve corresponds to $\varepsilon=0.1 \mathrm{~m}^{2} \mathrm{~s}^{-3}$, dotted curve $\varepsilon=1 \mathrm{~m}^{2} \mathrm{~s}^{-3}$, and dashed curve $-\varepsilon=10 \mathrm{~m}^{2} \mathrm{~s}^{-3}$.

One can see from Fig.5 that broadening dropsize distribution (increasing $\mu$ ) leads to decreasing DDV, and this phenomenon affects more if turbulence intensity is higher. These calculation results are improved by the investigation of median droplet diameter $D_{o}$ influence onto the DDV behavior. Median drop diameter is the second important parameter of Gamma dropsize distribution and can be reliably associated with rain rate. These results are given in Fig.6, where three curves represent different eddy dissipation rate values at the same $\mu=2$. 


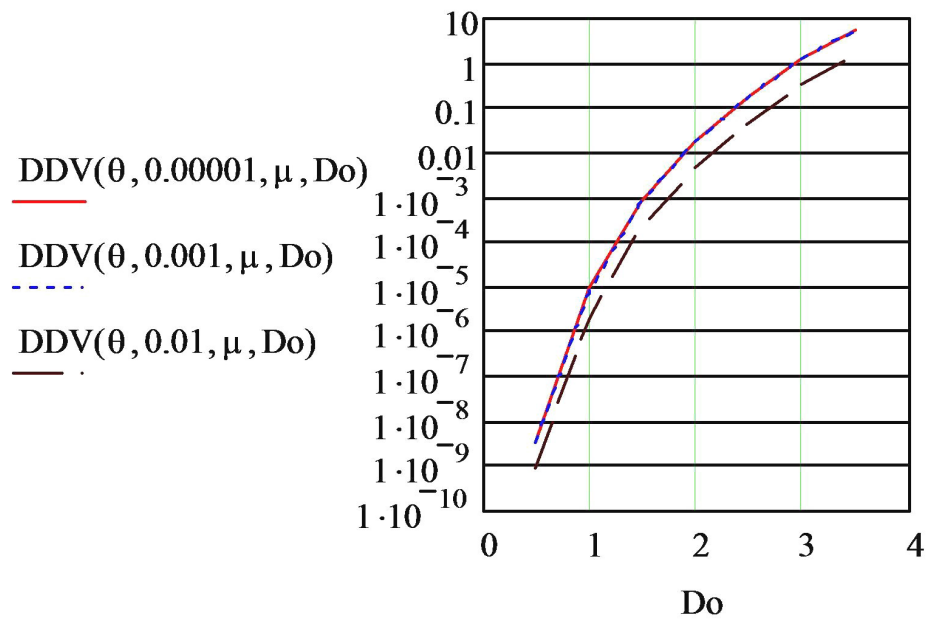

Fig.6. DDV curves as function of median drop diameter $D_{o}$ as a parameter of Gamma dropsize distribution model. Calculations were done at $\mu=2.0 \mathrm{~mm}$ and three values of eddy dissipation rate $\varepsilon$ indicated as numbers in $\left[\mathrm{m}^{2} \mathrm{~s}^{-3}\right]$. Solid curve corresponds to $\varepsilon=0.1$ $\mathrm{m}^{2} \mathrm{~s}^{-3}$, dotted curve $-\varepsilon=1 \mathrm{~m}^{2} \mathrm{~s}^{-3}$, and dashed curve $-\varepsilon=10 \mathrm{~m}^{2} \mathrm{~s}^{-3}$.

One can see that solid and dotted curves in Fig. 6 almost coincide. This fact means that light turbulence practically has quite minor impact on DDV as parameter that characterizes microstructure of the object under study.

\section{EXPERIMENTAL RESULTS}

Model validation as well as neural network algorithm check was done by using data acquired with the Transportable Atmospheric Radar (TARA) of the Delft University of Technology (TU-Delft), The Netherlands. TARA is S-band CW FM radar with high resolution, particularly the mode of $15 \mathrm{~m}$ range resolution was used during the observations of widespread and continuous rain in Cabauw Experimental Site for Atmospheric Research, located in the centre of the Netherlands.

Figure 7 shows measurement results of Doppler spectra from widespread rain at two orthogonal linear polarizations. One can see that horizontal polarization give larger values of maximum spectral components than vertical one. For convenience, the images of the spectra are mutually spaced in the picture.

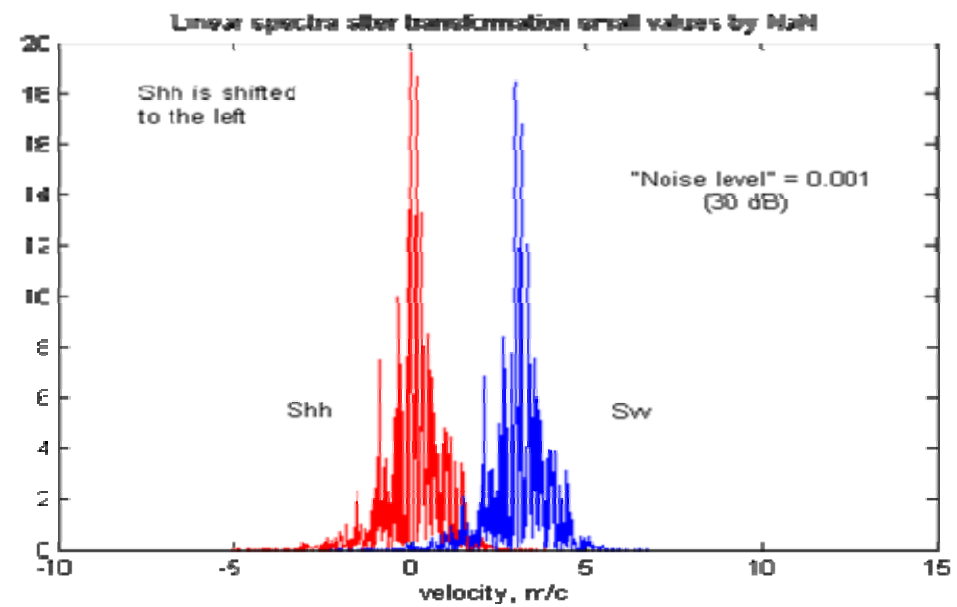

Fig.7. Measurement results: Doppler spectra at horizontal and vertical polarizations: reflections from widespread rain. The data taken in the Netherlands by IRCTR TARA radar system. 
The result of signal processing is presented in Fig. 8. The raw data were acquainted during 15 minutes and then processed to derive DDV in accordance with the theory given in section 2. For the comparison, in Fig. 9 the result of another processing of the same data is given to derive root mean square Doppler velocity that is often use to characterize turbulence intensity.

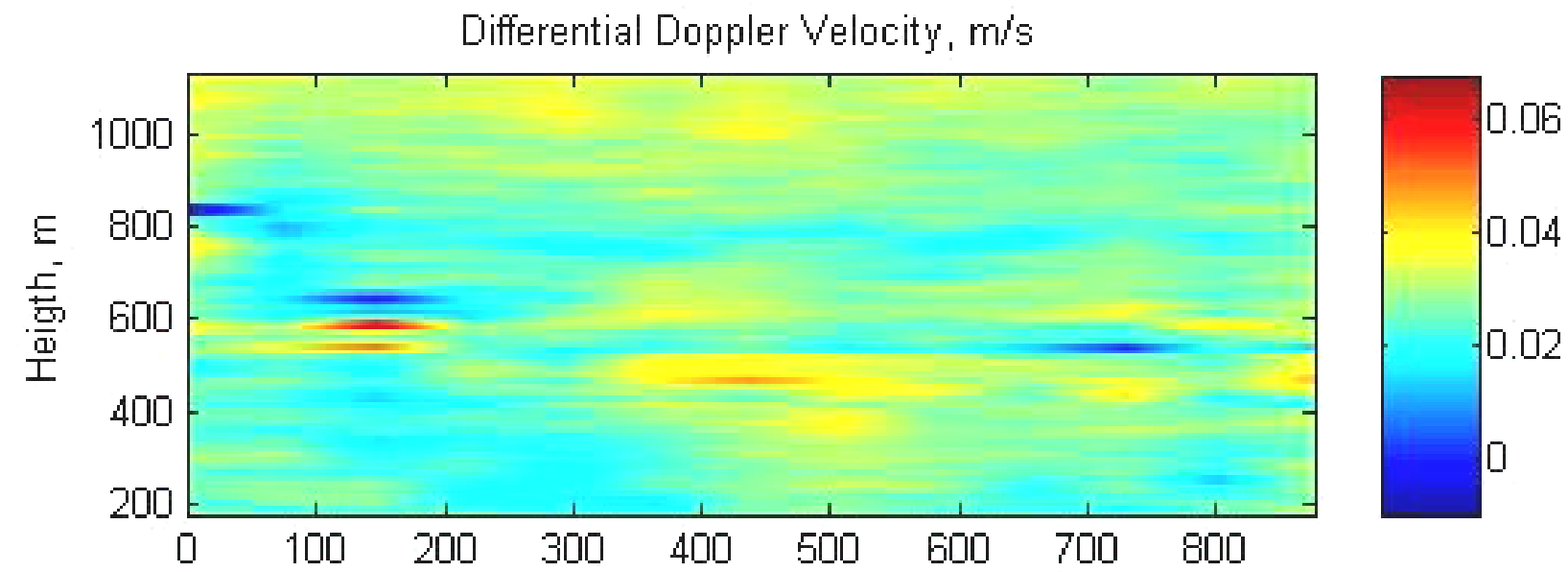

Fig.8. Measurement results: field of DDV in coordinates of "Height - Time". The time is in seconds. The color scale indicates DDV in $\mathrm{m} / \mathrm{s}$ from 0 to 0.07 .

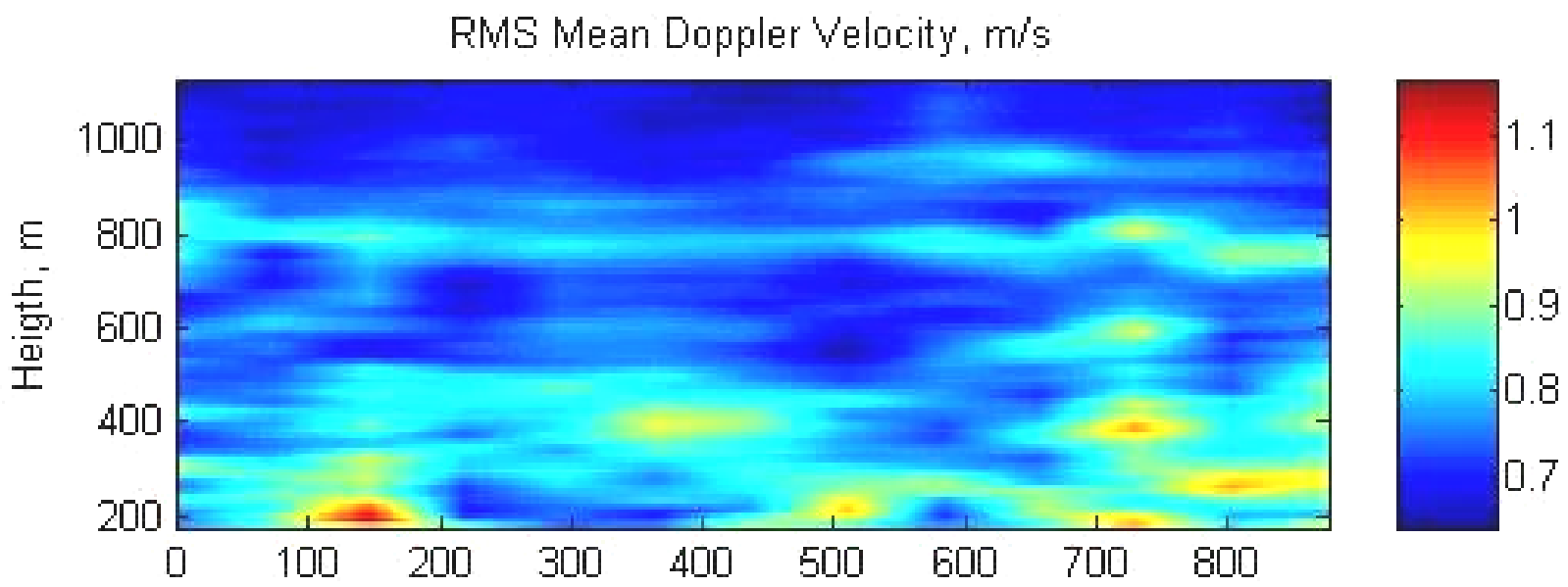

Fig.9. Measurement results: field of root mean square Doppler velocity in coordinates of "Height - Time". The time is in seconds. The color scale indicates DDV in $\mathrm{m} / \mathrm{s}$ up to $1.2 \mathrm{~m} / \mathrm{s}$.

Comparison of Fig. 8 and Fig. 9 allows coming to conclusion that DDV behavior in rain is on average opposite to rms Doppler velocity. It can be explain by the influence of turbulence that increases rms Doppler velocity and at the same time decreases DDV as was shown theoretically and by simulation in sections 3 and 4. Decreasing DDV is caused by addition of a random turbulent component to the vector of terminal drop fall velocity. 


\section{CONCLUSION}

In this paper, recently introduced Doppler-polarimetric parameter DDV defined as $\Delta V=\bar{V}_{h h}-\bar{V}_{v v}$, where $\bar{V}_{h h}$ and $\bar{V}_{v v}$ are mean Doppler velocities at horizontal and vertical polarization correspondingly has been studied theoretically and experimentally.

The relationship between DDV and parameters of microstructure of rain as an object of radar observation has been demonstrated theoretically. At the same time, it has been shown that DDV is affected by turbulent motions especially in case of strong turbulence. DDV is negatively correlated with turbulence.

Comparison of data processing results has proved that DDV and spectrum width parameters both can characterize turbulence in resolution volume.

What is especially important is independence of polarimetric parameters and Doppler parameters because they are based on different physics. That means that DDV can be considered as an additional source of information for remote sensing of atmosphere including neural network application to develop radar algorithms for automatic classification of hydrometeor type, turbulence intensity and especially dropsize distribution estimation in rain.

Influence of turbulence intensity on DDV value should be taken into account when using DDV as measurable parameter for deriving information about microstructure of rain. Moreover, it can be considered as one of measurands in the system estimating turbulence intensity and especially hydrometeor type recognitions.

\section{REFERENCES}

[1] F.J.Yanovsky, H.W.J. Russchenberg, L.P. Ligthart, and V.S. Fomichev, "Microwave Doppler-Polarimetric Technique for Study of Turbulence in Precipitation." Proc. IEEE IGARSS, Honolulu, USA, Vol. V, pp. 22962298 (2000).

[2] F.J. Yanovsky, and B.E. Fishman, "Mathematical model of processes of forming radar signals reflected from hydrometeors." Theory and Techniques of Radar, Navigation and Telecommunications, Riga, pp. 6-9 (1983). (in Russian).

[3] F.J. Yanovsky, H.W.J. Russchenberg, and C.M.H. Unal. "Retrieval of Information About Turbulence in Rain by Using Doppler-Polarimetric Radar", IEEE Transactions on Microwave Theory and Techniques, Feb., Vol. 53, No 2, pp. $444-450$ (2005).

[4] R. Wilson, A. J. Illingworth, and T. M. Blackman, "Differential Doppler Velocity: A Radar Parameter for Characterizing Hydrometeor Size Distributions," J. Appl. Meteor, 36, June, pp. 649-663 (1997).

[5] F.J. Yanovsky, Model of the relationship between Doppler and polarization parameters of the radar signal from precipitation. Report, Contract No IRCTR-S-002-98. TU-Delft, The Netherlands, January 1998, 108 pp.

[6] F. J. Yanovsky, "Phenomenological Models of Doppler-Polarimetric Microwave Remote Sensing of Clouds and Precipitation," IEEE IGARSS, Toronto, Canada, Vol. 3, pp. 1905 -1907 (2002).

[7] F.J. Yanovsky, C.M.H. Unal, and H.W.J. Russchenberg. Relationship between Differential Doppler Velocity and Turbulence Intensity in Rain: Modeling and Measurements. Proceedings 6th International Symposium on Tropospheric Profiling ISTP 2003, Leipzig, Saxony. Extended Abstracts, pp. 306-308 (2003).

[8] F.J. Yanovsky, A.A. Pitertsev, Y.P. Ostrovsky et al, "Neural Network Identification Algorithm for Weather Radar." Extended Abstracts ISTP7, 2006, Boulder, Co, USA, paper 6.2, 3 pp. (2006).

[9] D. Atlas, R. C. Srivastava, and R. S. Sekhon, "Doppler radar characteristics of precipitation at vertical incidence," Reviews of Geophysics and Space physics, vol. 11, no 1 (1973). 